\title{
Representações Sociais de usuários sobre Doenças Negligenciadas
}

\author{
Social Representations of users about Neglected Diseases \\ Representaciones Sociales de los usuarios sobre Enfermedades Desatendidas
}

Recebido: 09/03/2021 | Revisado: 16/03/2021 | Aceito: 19/03/2021 | Publicado: 27/03/2021

Charles Souza Santos

ORCID: https://orcid.org/0000-0001-5071-0359

Universidade Estadual do Sudoeste da Bahia, Brasil

E-mail:charlesss@uesb.edu.br

Antonio Marcos Tosoli Gomes

ORCID: https://orcid.org/0000-0003-4235-9647

Universidade do Estado do Rio de Janeiro, Brasil

E-mail: mtosoli@gmail.com

Anadir de Almeida Farias

ORCID: https://orcid.org/0000-0002-2124-531X

Universidade Estadual do Sudoeste da Bahia, Brasil

E-mail: nnhfarias@gmail.com

Maicla Oliveira da Silva

ORCID: https://orcid.org/0000-0002-4536-2262

Universidade Estadual do Sudoeste da Bahia, Brasil

E-mail: maicla64@gmail.com

Rebecca Calheira Barreto

ORCID: https://orcid.org/0000-0001-7555-6714

Universidade Estadual do Sudoeste da Bahia, Brasil

E-mail: rebeccacalheira@gmail.com

Marcela Rossi Ribeiro

ORCID: https://orcid.org/0000-0002-7874-6086

Universidade Estadual do Sudoeste da Bahia, Brasil

E-mail: marcelarossiribeiro@gmail.com

Marcio Pereira Lobo

ORCID: https://orcid.org/0000-0002-8454-2135

Universidade Estadual do Sudoeste da Bahia, Brasil

E-mail: marcioplobo@gmail.com

Gleide Magali Lemos Pinheiro

ORCID: https://orcid.org/0000-0002-0848-3348

Universidade Estadual do Sudoeste da Bahia, Brasil E-mail: gleidemlp@gmail.com

Flávia Silva Souza

ORCID: https://orcid.org/0000-0002-8994-4117 Universidade Estadual do Sudoeste da Bahia, Brasil E-mail:flaviameg@gmail.com

Lyra Cândida Calhau Rebouças

ORCID: https://orcid.org/0000-0003-0576-4360

Universidade Estadual do Sudoeste da Bahia, Brasil E-mail:lyracalhau@gmail.com

\begin{abstract}
Resumo
Objetivo: Analisar a estrutura das representações sociais de usuários dos serviços de saúde sobre doenças negligenciadas. Metodologia: estudo qualitativo sustentado na Teoria das Representações Sociais, em sua abordagem estrutural, composto por amostra intencional e não-probabilística de 126 usuários dos serviços de saúde. Dados coletados por questionário de evocações livres ao termo indutor doenças negligenciadas e analisados pela técnica do quadrante de quatro casas, auxílio do software Ensemble de Programmes Permettant l'analyse dês Evocations 2005 e pela análise de similitude. Resultados: Evidenciaram uma estrutura representacional de usuários dos serviços de saúde sobre doenças negligenciadas constituída por três dimensões: individual, social e imagética, que foram gerenciadas por um núcleo central formado pelos cognemas descaso e ignorância. As dimensões e o núcleo central desta estrutura representacional apresentaram conhecimentos socialmente elaborados pelos participantes e os seus significados sobre a prevenção, o controle e o tratamento destas enfermidades. Conclusão: A estrutura representacional implicou na construção de práticas colaborativas dos usuários dos serviços de saúde para elaboração de estratégias de enfrentamento às doenças negligenciadas.
\end{abstract}

Palavras-chave: Doenças negligenciadas; Serviços de saúde; Prevenção de doenças. 


\begin{abstract}
Objective: To analyze the structure of social representations of users of health services about neglected diseases. Methodology: qualitative study based on the Theory of Social Representations, in its structural approach, composed of an intentional and non-probabilistic sample of 126 users of health services. Data collected by questionnaire of free evocations to the term inducer neglected diseases and analyzed by the technique of the quadrant of four houses, aided by the software Ensemble of Programs Permettant l'analyse DES Evocations 2005 and by the similarity analysis. Results: They evidenced a representational structure of users of health services on neglected diseases, constituted by three dimensions: individual, social and imagery, which were managed by a central nucleus formed by the neglect and ignorance cognemas. The dimensions and central core of this representational structure presented knowledge socially elaborated by the participants and their meanings about the prevention, control and treatment of these diseases. Conclusion: The representational structure implied the construction of collaborative practices by users of health services to develop strategies to cope with neglected diseases.
\end{abstract}

Keywords: Neglected diseases; Health services; Prevention of diseases.

\title{
Resumen
}

Objetivo: Analizar la estructura de las representaciones sociales de los usuarios de los servicios de salud sobre las enfermedades desatendidas. Metodología: estudio cualitativo basado en la Teoría de las Representaciones Sociales, en su enfoque estructural, compuesto por una muestra intencional y no probabilística de 126 usuarios de los servicios de salud. Datos recogidos mediante cuestionario de evocaciones libres al término inductor de enfermedades desatendidas y analizados mediante la técnica del cuadrante de cuatro casas, con la ayuda del software Ensemble of Programs Permettant l'analyse DES Evocations 2005 y por el análisis de similitud. Resultados: evidenciaron una estructura representativa de los usuarios de los servicios de salud sobre enfermedades desatendidas, constituida por tres dimensiones: individual, social e imaginaria, las cuales fueron manejadas por un núcleo central formado por los cognemas de abandono e ignorancia. Las dimensiones y núcleo central de esta estructura representativa presentaron conocimientos elaborados socialmente por los participantes y sus significados sobre la prevención, control y tratamiento de estas enfermedades. Conclusión: La estructura representacional implicó la construcción de prácticas colaborativas por parte de los usuarios de los servicios de salud para desarrollar estrategias de afrontamiento de enfermedades desatendidas.

Palabras clave: Enfermedades desatendidas; Servicios de salud; Prevención de enfermedades.

\section{Introdução}

As Doenças negligenciadas (DN) são causadas por agentes infecciosos ou parasitas, que além de predominar em situações de pobreza, também colaboram para a continuidade do cenário de desigualdade, visto que propicia barreiras para o progresso dos países. Dessa forma, as DN são deixadas de lado por não possuírem atrativos econômicos para o desenvolvimento de fármacos e pesquisas, seja por sua taxa reduzida de prevalência em países desenvolvidos, ou por atingir populações menos favorecidas. No rol das DD destacam-se: leishmaniose, malária, dengue, tuberculose, doença de Chagas, esquistossomose, hanseníase, dentre outras (Ministério da saúde, 2010; Resolução RDC nº 28, 2007).

As DN são endêmicas em 149 países e acometem mais de um bilhão de pessoas no mundo. Muitas destas doenças são fatais ou geram consequências incapacitantes, ocasionando implicações nos âmbitos familiar, econômico e social como, também, sobrecarregam os sistemas de saúde de países já economicamente desfavorecidos, e comprometem a capacidade produtiva de sua população, formando um ciclo vicioso de alta repercussão no desenvolvimento humano, com forte predomínio da exclusão social e acentuação de estigmas historicamente criados que reduzem perspectivas futuras de gerações (World Health Organization, 2016; Morel, 2006).

No Brasil, logo após a descentralização das condutas de controle endêmico para a esfera municipal, diversas doenças negligenciadas deixaram de ser priorizadas, ocasionando elevação da incidência de algumas dessas doenças e de focos de contaminação por vetores e/ou hospedeiros intermediários. Diante desse cenário, faz-se necessário intensificação na vigilância da epidemiologia das DN, visto que estas têm consequências progressivas, como supracitado (Santos et al., 2017).

Em nível mundial, o controle das DN tem sido essencialmente vertical. Destarte, faz-se relevante investigar como pode ser feita a associação para a prevenção e controle dessas doenças na comunidade. Análises econômicas podem ser úteis para prever as consequências financeiras de qualquer mudança no método de implementação e no investimento para descoberta e produção de 
insumos medicamentos, em conformidade com o desenvolvimento tecnológico que a ciência vivencia no século atual. Além disso, a análise dos níveis de financiamento viáveis para garantir a cobertura elevada das intervenções para controle das doenças negligenciadas é importante para os países e as organizações internacionais, com o objetivo de garantir a sustentabilidade e continuidade dessas ações (Conteh \& Molyneux, 2010).

Em razão disso, esta pesquisa enfoca a compreensão de usuários dos serviços de saúde em face das doenças negligenciadas, uma vez que este tema merece atenção por causar principalmente, sofrimento, incapacidade permanente e morte nas populações que já vivem em situação de extrema pobreza. Sendo assim, a compreensão desses usuários sobre tal temática pode ser investigada à luz da Teoria das Representações Sociais (TRS), que são modelos de conhecimento prático guiadas para a comunicação e para o entendimento do contexto social, material e ideativo no qual vivemos. Em outras palavras, a TRS aborda os saberes sociais, a partir daí, como esses saberes orientam o pensamento, as ações e por conseguinte, as formas de agir dos indivíduos na sociedade (Spink, 1993).

Os conhecimentos e saberes elaborados pelos usuários dos serviços de saúde certamente favorecem a compreensão das atitudes e práticas para o enfrentamento das doenças negligenciadas. As representações sociais são modalidades de pensamento prático orientado para a comunicação, a compreensão e o domínio do ambiente social, material e ideal (Spink, 1993).

Esse estudo tem por objetivo analisar a estrutura das representações sociais de usuários dos serviços de saúde a respeito das doenças negligenciadas.

\section{Metodologia}

Trata-se de uma pesquisa de natureza qualitativa com sustentação na Teoria das Representações Sociais em sua abordagem estrutural, com intuito de analisar e discutir os significados atribuídos pelos usuários dos serviços de saúde frente às doenças negligenciadas. As abordagens qualitativas se caracterizam pela preocupação em compreender a maneira de agir e pensar das pessoas ou dos grupos e procura responder às exigências colocadas pelos fenômenos estudados. Ela pode ser aplicada em diferentes dimensões, como a vida de um grupo, suas estruturas de pensamento, suas crenças, seus códigos de conduta, seus costumes e as experiências vivenciadas no cotidiano pelas pessoas. (Jodelet, 2003).

Para Pereira et al (2018), nos métodos qualitativos é importante que o pesquisador interprete com suas opiniões acerca do fenômeno estudado. Neste tipo de metodologia, a coleta de dados ocorre na maioria das vezes através de entrevistas nas quais as questões são abertas.

A amostra foi do tipo intencional, não probabilística, composta por 126 usuários dos serviços de saúde de uma população de 432, que foram assistidos na rede de serviços de saúde no município de Jequié/BA. Os critérios de inclusão da amostra foram: (1) usuários dos serviços de saúde cadastrados nas unidades de saúde e que portavam o diagnóstico de pelo menos uma das doenças consideradas negligenciadas no Brasil; (2) Os critérios de exclusão foram: usuários dos serviços de saúde que não são portadores das doenças negligenciadas.

Os dados foram coletados através da aplicação da técnica de evocação livre, no período de janeiro a abril de 2019, que consistiu em solicitar aos participantes que evocassem imediatamente 05 palavras ou expressões ao termo indutor "doenças negligenciadas". As palavras ou termos foram registrados em formulário próprio na ordem em que foram mencionadas. Os participantes foram recrutados nas unidades primárias e secundárias de saúde, no município de Jequié, Bahia. A aplicação da técnica demandou aproximadamente 15 minutos para a sua realização.

A técnica de evocações livres é considerada como uma técnica maior para coletar os elementos constitutivos do conteúdo de uma representação. Ela consiste em pedir aos sujeitos que, a partir de um termo indutor (normalmente, o próprio rótulo verbal que designa o objeto da representação) apresentado pelo pesquisador, digam as palavras ou expressões que lhes 
tenham vindo imediatamente a lembrança (Abric, 2001). Os vocábulos provenientes da técnica de evocações foram organizados em documento word, que constituiu o corpus de análise.

O tratamento dos dados se deu pelo software Ensemble de Programmes Permettant l'analyse dês Evocations (EVOC), versão 2005, que analisa estatisticamente os dados textuais de uma rede associativa, na qual é permitido combinar a frequência de aparição de palavras evocadas com a atribuição de sua ordem de importância (Vergés, 2002). Os dados foram submetidos a análise prototípica e análise de similitude por coocorrência.

A análise prototípica (também chamada análise de evocações ou das quatro casas) é uma das técnicas mais difundidas para caracterização estrutural de uma representação social. Essa técnica constitui-se de duas etapas: a primeira, chamada análise prototípica, baseia-se no cálculo de frequências e ordens de evocação das palavras, enquanto que uma segunda etapa se centra na formulação de categorias englobando as evocações e avalia suas frequências, composições e coocorrências (Walchelke \& Wolter, 2011).

A análise foi realizada pela técnica do quadro de quatro casas (análise prototípica) com o intuito de identificar o possível núcleo central das representações sociais. A combinação desses dois critérios, frequência de evocação e ordem média de evocação de cada palavra, possibilita assim o levantamento daquelas que mais provavelmente pertencem ao núcleo central da representação, por seu caráter prototípico, ou, por sua saliência. Na técnica, a partir da interseção da frequência média de evocação do inteiro conjunto de palavras com a média de suas respectivas ordens médias de evocação, são definidos quatro quadrantes que conferem diferentes graus de centralidade às palavras que os compõem (Vergés, 1994).

A análise de similitude envolve a partir de um conjunto de pares pareados ou agrupados, o cálculo de um índice de similitude entre cada par de itens. O mais simples destes índices consiste na relação entre o número de coocorrências (número de ligações estabelecidas entre dois itens específicos) e o número de sujeitos envolvidos. Ou admite-se que dois itens serão tanto mais próximos na representação quanto um número mais elevado de sujeitos os trate da mesma maneira (Sá, 2002).

A partir da análise prototípica foram utilizados os cognemas apresentados no quadro de quatro casas para a elaboração da árvore máxima. Realizando essa operação para cada par de itens, vai se dispor da matriz de similitude para todos os itens do corpus. Para facilitar a compreensão e a interpretação de tais matrizes, constrói-se a "árvore máxima". Trata-se de um grafo conexo sem ciclo cujos vértices são os itens do corpus e as arestas são os valores dos índices de similitude entre esses itens. O procedimento de construção da árvore máxima permite reter apenas as relações mais fortes entre os itens (Moliner, 1994).

A pesquisa obedeceu aos critérios éticos preconizados pela Resolução no 466 de 12 de dezembro de 2012, do Conselho Nacional de Saúde. O projeto foi aprovado pelo Comitê de Ética em Pesquisa (CEP) da Universidade do Estadual do Sudoeste da Bahia (UESB) em 05 de junho de 2017, com número de protocolo n ${ }^{\circ} 2.113 .727$, sendo obtida autorização da Secretaria Municipal de Saúde de Jequié, além da autorização dos participantes, expresso na assinatura do Termo de Consentimento Livre e Esclarecido.

\section{Resultados}

O grupo de usuários dos serviços de saúde foram caracterizados pelas variáveis que influenciaram a construção simbólica e o imaginário social sobre as doenças negligenciadas. Os participantes que formaram este grupo representacional no total de 126 usuários dos serviços de saúde foram em sua maioria 56,3\% do sexo masculino; possuíam idade entre 18 a 65 anos; com diagnóstico médico das seguintes doenças negligenciadas: 10 com tuberculose pulmonar; 06 com hanseníase; 17 com leishmanioses; 81 com esquistossomose e 12 com doença de Chagas.

Com relação à estrutura da evocação, destaca-se que o conjunto dos sujeitos evocaram 564 palavras, sendo 94 palavras diferentes, gerando um quadro de quatro casas com uma frequência mínima de 12 , frequência média de 23 e a ordem das ordens médias de 2,6. Expõe-se, a seguir, o Quadro 1 conforme o resultado elaborado pelo software EVOC 2005. 
Quadro 1 - Quadro de quatro casas ao termo indutor "doenças negligenciadas" entre usuários dos serviços de saúde. Jequié/BA, $2018(n=126)$.

\begin{tabular}{|c|c|c|c|c|c|c|}
\hline \multicolumn{4}{|c|}{ O.M.E. $<2,60$} & \multicolumn{3}{|l|}{$>2,60$} \\
\hline $\begin{array}{l}\text { Freq. } \\
\text { Med. }\end{array}$ & Termo evocado & Freq. & O.M.E. & Termo evocado & Freq. & O.M.E. \\
\hline$\geq 23$ & $\begin{array}{l}\text { Descaso } \\
\text { Ignorância }\end{array}$ & $\begin{array}{l}30 \\
38\end{array}$ & $\begin{array}{l}2,20 \\
2,52\end{array}$ & $\begin{array}{l}\text { Câncer } \\
\text { Leishmania } \\
\text { Tuberculose } \\
\text { Schistosoma }\end{array}$ & $\begin{array}{l}24 \\
38 \\
42 \\
42\end{array}$ & $\begin{array}{l}2,91 \\
2,84 \\
3,52 \\
2,71\end{array}$ \\
\hline$\leq 12$ & $\begin{array}{l}\text { Dor } \\
\text { Falta-assistência }\end{array}$ & $\begin{array}{l}14 \\
18\end{array}$ & $\begin{array}{l}2,28 \\
2,33\end{array}$ & $\begin{array}{l}\text { Chagas } \\
\text { Doenças } \\
\text { Falta-investimento } \\
\text { Hanseníase } \\
\text { Mal-governo } \\
\text { Pobreza } \\
\text { Tratamentos }\end{array}$ & $\begin{array}{l}18 \\
12 \\
12 \\
18 \\
14 \\
18 \\
12\end{array}$ & $\begin{array}{l}3,33 \\
3,00 \\
2,83 \\
2,88 \\
3,14 \\
3,00 \\
3,83 \\
\end{array}$ \\
\hline
\end{tabular}

Fonte: Arquivos da pesquisa.

O Quadro 1 apresenta o quadrante de quatro casas que indica a possível estrutura das representações sociais dos usuários dos serviços de saúde sobre as doenças negligenciadas. O quadrante superior esquerdo indica o possível núcleo central enquanto os demais quadrantes representam o sistema periférico da estrutura representacional.

As palavras que compuseram o quadrante de quatro casas num total de 15 são definidas como cognemas que formam o pensamento psicossocial dos participantes deste estudo e definem a estrutura e o conteúdo das representações sociais. Em termos mais técnicos, a representação social é composta por cognemas que se relacionam entre si e formam um conjunto que se transforma a partir de regras que preservam a totalidade. O cognema é o elemento cognitivo básico, em outros termos, a menor unidade da cognição neste nível de análise. Uma representação é então composta por ideias (ou cognemas ou elementos) que são ativadas quando um grupo pensa um objeto. Ao pensar o objeto muitas ideias são ativadas mas nem todas são equivalentes, alguns têm mais valor que outros (Wolter, 2016).

A partir dos dados decorrentes da análise prototípica foi possível determinar o grau de conexidade destes elementos a partir da construção da árvore máxima considerando a coocorrência destes cognemas entre os sujeitos envolvidos neste estudo. Nesta análise de similitude gerou-se a árvore máxima que segue abaixo na Figura 1: 
Figura 1 - Árvore máxima de similitude por coocorrência das evocações dos usuários dos serviços de saúde ao termo indutor doenças negligenciadas - Jequié/BA, 2018 ( $\mathrm{n}=126)$.

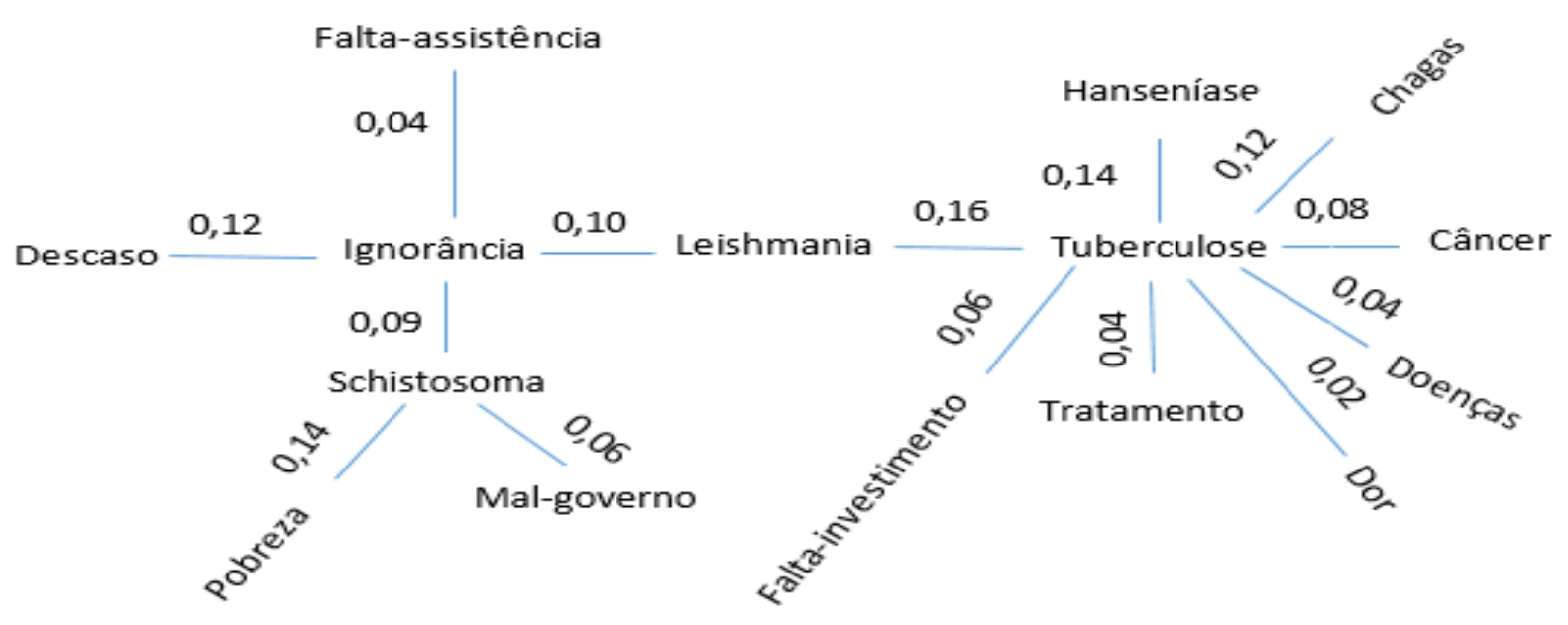

Fonte: Arquivos da pesquisa.

$\mathrm{Na}$ árvore máxima identifica-se o maior número de conexões dos elementos ao cognema tuberculose com 08 ligações; em segundo, ignorância com 04 ligações e, finalmente, a schistosoma com 03 ligações. Sobre a relação entre os cognemas representacionais pode-se afirmar que esta análise favorece a ideia de centralidade para os termo ignorância, pois é o cognema que possui maior conexidade com os demais elementos que compõem a estrutura representacional.

\section{Discussão}

Os cognemas apresentados no quadrante de quatro de casas constituem a possível estrutura representacional do pensamento psicossocial dos usuários dos serviços de saúde. Os cognemas que se alocaram no quadrante superior esquerdo, o descaso e a ignorância, formam o possível núcleo central enquanto os demais quadrantes são construtores do sistema periférico. Abric (1998) sustenta a hipótese de que toda representação social está organizada em torno de um núcleo central e um sistema periférico. Assim, a representação social constitui-se num conjunto organizado e estruturado de informações, crenças, opiniões e atitudes, composta de dois subsistemas - o central e o periférico -, que funcionam exatamente como uma entidade, onde cada parte tem um papel específico e complementar (Machado \& Aniceto, 2010).

Para Abric (1994, p.19) a Representação Social possui uma estrutura pois é constituída por um conjunto de cognemas que são organizados e de status diferenciado, mais especificamente para o autor "os elementos que constituem uma representação são hierarquizados, ponderados e mantêm, entre si, relações que determinam o significado, e o lugar que ocupam no sistema representacional". A representação social pode, em consequência, ser concebida como um conjunto de ideias, que se relaciona entre si, pensada por um grupo acerca de um objeto.

O núcleo central está relacionado com a memória coletiva traduzida na significação, consistência e permanência da representação sendo, portanto, estável e resistente a mudanças (Machado \& Aniceto, 2010). Neste contexto, Abric (1994), sustentou-se na elaboração de uma estrutura para a representação social formada por um núcleo central e elementos periféricos onde a sua Organização Estrutural reflete o diferencial entre representações. O núcleo central apresenta-se estável e resistente às mudanças, tradutor de significação, consistência e permanência na representação, relacionado com a memória coletiva.

O cognema descaso sugere o entendimento de que os usuários dos serviços de saúde atribuem as causas e perpetuação das doenças negligenciadas a ausência de práticas individuais capazes de evitar a instalação destas morbidades. O descaso com a 
própria saúde reflete a dimensão individual dos conteúdos desta representação social uma vez que, o primeiro passo para o adoecimento do corpo é a ausência do autocuidado.

O cognema ignorância, por sua vez, reflete a ausência das informações e conhecimentos por parte dos usuários dos serviços de saúde sobre os aspectos que englobam as doenças negligenciadas, como por exemplo, os fatores biomédicos e os possíveis determinantes sociais em saúde que não fazem parte do imaginário social e tão pouco do senso comum. São esses elementos que giram em torno do indivíduo e da sociedade que definem a instalação ou prevenção destas entidades mórbidas.

No quadrante superior direito os cognemas que representam a dimensão imagética das doenças negligenciadas no pensamento psicossocial dos usuários dos serviços de saúde foram apresentados: câncer, leishmania, tuberculose e schistosoma. Entre essas doenças somente o câncer não é considerado como negligenciada conforme definições das instituições de saúde.

Em 2005, o Ministério da Saúde do Brasil lançou um programa de pesquisa e desenvolvimento em doenças negligenciadas que incluía seis doenças: dengue, doença de Chagas, leishmanioses, hanseníase, malária e tuberculose. Já no "Relatório Saúde Brasil 2017: uma análise da situação de saúde e os desafios para o alcance dos Objetivos de Desenvolvimento Sustentável”, o Ministério da Saúde listou as seguintes doenças como negligenciadas: doença de Chagas, esquistossomose mansoni, hanseníase, filariose linfática, leishmaniose tegumentar, leishmaniose visceral, oncocercose, raiva humana, tracoma (Secretaria de Vigilância em Saúde, 2018).

Esses cognemas foram citados frequentemente pelos participantes pois essas doenças fazem parte do seu cotidiano e tais pessoas convivem com suas mazelas e desafios no enfrentamento de todos os danos sejam físicos ou emocionais ocasionados pelos estigmas e preconceito que entornam as doenças negligenciadas.

O quadrante inferior esquerdo traz a discussão dos significados atribuídos aos aspectos biológicos das doenças negligenciadas por meio do cognema dor e ainda sobre as dificuldades na realização do tratamento com a evocação do cognema falta-assistência.

O quadrante inferior direito formado pelos cognemas chagas, doenças, falta-investimento, hanseníase, mal-governo, Pobreza, Tratamentos. Os cognemas pobreza, mal-governo e falta-investimento, que se destacaram por sua saliência no quadrante de quatro casas, compreendem uma dimensão que está intimamente relacionada ao determinismo social e individual, pois são colocados como principais causadores das doenças negligenciadas. A pobreza e a miséria abrangem grande parte da população mundial e apesar das ações estratégicas de algumas instituições, ainda não foram totalmente superadas.

O termo pobreza possui um significado singular na representação das doenças negligenciadas, pois determina e desencadeia todo o processo de vulnerabilidade social do indivíduo, causada pela ausência de estratégias econômicas dos países para diminuir as desigualdades sociais. As doenças negligenciadas existem porque a pobreza existe, por isso as ações governamentais que diminuem as desigualdades sociais precisam ser fortalecidas, principalmente no Brasil, onde ainda se convive em um cenário de extrema pobreza e, não por acaso, assume a maior carga de doenças negligenciadas da América Latina e Caribe. Isto significa que grande parte do contingente de 40 milhões da população mais pobre do Brasil está infectada por uma ou mais doenças negligenciadas (Santos et al., 2017).

Para confirmação da centralidade do provável núcleo central das RS e informação do grau de conexidade entre os elementos do sistema central (SC) e periférico (SP), a escolha da análise de similitude tornou-se fundamental para a compreensão dos agrupamentos e conexões entre os elementos identificados, indicando conforme, Alves Mazzotti (2007, p.299), que a organização dos elementos da representação, nos ajuda a compreender os sentidos dos termos

$\mathrm{Na}$ árvore máxima os cognemas tuberculose, ignorância e schistosoma tiveram ligações a outros cognemas com mais frequência e significam a confirmação ou refutação dos termos centrais desta representação social. O cognema tuberculose reflete a dimensão imagética das representações sociais confirmando a elaboração de conhecimentos dos usuários dos serviços de saúde 
relacionados ao convívio com as doenças negligenciadas e o seu enfrentamento buscando o tratamento medicamentos e outras alternativas plausíveis no contexto social.

O cognema ignorância agrega os valores voltados para a prática socioindividual diante das doenças negligenciadas e a necessidade de ampliar os conhecimentos dos usuários a respeito das formas de prevenção e de sua participação nas políticas de saúde que favoreçam o controle e o combate a esses agravos. O termo ignorância também aparece como constituinte do possível núcleo central desta representação social.

\section{Conclusão}

O estudo possibilitou a identificação da estrutura da representação social dos usuários dos serviços de saúde acerca das doenças negligenciadas. Deste modo, foi descrito um núcleo central que organiza a representação, assim como um sistema periférico que o sustenta.

A estrutura representacional se organizou ao redor dos léxicos descaso e ignorância, que permearam a descrição das principais questões ligadas às doenças negligenciadas constituindo as dimensões individual, social e imagética. Cada dimensão apresentou conhecimentos socialmente elaborados pelos participantes e os seus significados sobre a prevenção, o controle e o tratamento destas entidades mórbidas.

A estrutura representacional implicou na construção de práticas colaborativas dos usuários dos serviços de saúde para elaboração de estratégias de enfrentamento às doenças negligenciadas. Por fim, ressalta-se a necessidade da realização de mais estudos sobre a temática em questão para melhor compreensão e ainda, para alicerçar o desenvolvimento de políticas de saúde voltadas para as doenças negligenciadas.

\section{Referências}

Abric, J. C. (2003). Abordagem estrutural das representações sociais: desenvolvimentos recentes. In: Campos PHF, Loureiro MCS. Loureiro. Representações sociais e práticas educativas. Goiânia: UCG.

Abric, J. C. (1998). A abordagem estrutural das representações sociais. In A. S. Moreira \& D. C. Oliveira (Eds.), Estudos interdisciplinares de representação social. Goiânia: Ed. AB.

Abric, J. C. (2001) Práticas sociales y representaciones. Ciudad de México: Ediciones Coyoacán.

Abric, J. C. (1994). Pratiques sociales et representations. PUF.

Conteh, L., Engels, T., \& Molyneux, D. H. (2010). Socioeconomic aspects of neglected tropical diseases. The Lancet. 375 (9710), $239-247$.

Ministério da Saúde. (2010). Doenças negligenciadas: estratégias do Ministério da Saúde. Rev Saúde Pública, 44 (1), $200-222$.

Jodelet, D. (2003). Os métodos das ciências humanas. In: Moscovici S, Buschini F. Olhares sobre as metodologias qualitativas. Paris: PUF. 139-162

Machado, L. B., \& Aniceto, R. (2010). Núcleo Central e periferia das representações sociais de ciclos de aprendizagem entre professores. Ensaio: Avaliação e Políticas Públicas em Educação, 345- 364.

Mazzotti, A. J. (2002). A abordagem estrutural das representações sociais. Psicologia da Educação, $1^{\circ}$ e $2^{\circ}$ semestre, $17-37$.

Moliner, P. (1994). L'etude experimentale des processus representattionels. Commentaire de l'article de R. Michit. Papers on Social Representations. 118-122.

Morel, C. M. Inovação em saúde e doenças negligenciadas (Editorial) (2006). Cad Saude Publica. 22 (8), $1522-1523$.

Pereira, A. S., Shitsuka, D. M., Pereira, F. J., \& Sitsuka, R. (2018). Metodologia da pesquisa científica [recurso eletrônico], UFSM.

Resolução RDC n 28, de 4 de abril de 2007. (2007). Dispõe sobre a priorização da análise técnica de petições, no âmbito da Gerência-Geral de Medicamentos da ANVISA, cuja relevância pública se enquadre nos termos desta Resolução. Brasil. www.diariodasleis.com.br/busca/exibelink.php?numlink=1-9-34-200704-04-28.

Sá, C. P. (2002). Núcleo central das representações sociais. Vozes.

Secretaria de Vigilância em Saúde. (2018). Doenças negligenciadas no Brasil: vulnerabilidade e desafios. In BRASIL. Ministério da Saúde. Brasil 2017. Uma análise da situação de saúde e os desafios para o alcance dos Objetivos de Desenvolvimento Sustentável. Brasília: Ministério da saúde. Recuperado em bvsms.saude.gov.br/bvs/publicacoes/saude_brasil_2017_analise_situacao_saude_desafios_objetivos_desenvolvimento_sustetantavel.pdf. 
Research, Society and Development, v. 10, n. 3, e56810313708, 2021

(CC BY 4.0) | ISSN 2525-3409 | DOI: http://dx.doi.org/10.33448/rsd-v10i3.13708

Santos, C. S., Gomes, A. M. T., Souza, F. S., Marques, S. C., Lobo, M. P., \& Oliveira, D. C. (2017). Representações sociais de profissionais de saúde sobre doenças negligenciadas. Esc Anna Nery. 21 (1).

Santos, L. P., Nogueira, M. J., Rezende, C. N., \& Ferreira, R. A. (2017). Doenças negligenciadas no município de Sabará: casos, portadores e percepções. Arq. Cienc. Saúde UNIPAR. 21(3), 155-162.

Spink, M. J. P. (1993). O Conceito de Representação Social na Abordagem Psicossocial. Cad. Saúde Públ. 9 (3), 300-308.

Vergés, P. (1994). Approche du noyau central: proprieties quantitatives et estructurales. In: Guimelli C. Structures et transformations des representations sociales. Neuchâtel, Delachaux et Niestlé. 233-253.

Vergés, P. (2002). Ensemble de programmes permettant l'abalyse des evocatios - Manuel. Version.

Wachelke, J., \& Wolter, R. (2011). Critérios de construção e relato da análise prototípica para representações sociais. Psic.: Teor. e Pesq. Brasília. 27(4), 521526.

Wolter, R. P., Wachelke, J., \& Naiff, D. (2016). A abordagem estrutural das representações sociais e o modelo dos esquemas cognitivos de base: perspectivas teóricas e utilização empírica. Temas psicol. 24(3), 1139-1152.

World Health Organization/WHO. (2016). Neglected tropical diseases.www.who.int/neglected_diseases/diseases/en/. 